\title{
Severe maternal outcome: a review
}

\section{Suparna Grover, Harbhajan Kaur Shergill, Ajay Chhabra*}

\author{
Department of Obstetrics and Gynaecology, Government Medical College, Baba Farid University of Health Sciences, \\ Amritsar, Punjab, India
}

Received: 14 January 2016

Accepted: 08 February 2016

\author{
*Correspondence: \\ Dr. Ajay Chhabra, \\ E-mail: drajaychhabra@gmail.com
}

Copyright: (C) the author(s), publisher and licensee Medip Academy. This is an open-access article distributed under the terms of the Creative Commons Attribution Non-Commercial License, which permits unrestricted non-commercial use, distribution, and reproduction in any medium, provided the original work is properly cited.

\begin{abstract}
Maternal mortality ratio (MMR) is considered an indicator of obstetric care available in a society and reduction in MMR has been one of the important millennium development goals defined by World Health Organization (WHO) but it has always been recognized that maternal mortality is just the tip of iceberg. WHO has now defined maternal near-miss cases thus broadening the focus on life threatening conditions encountered by pregnant women. A study was started at our obstetric unit based on near-miss maternal mortality concept in March 2015 and is currently underway. We analyzed the initial data of the first few months and recognized four of such special cases which find mention in this review. We decided to review the literature with special reference to these cases as every such case is a lesson in itself for the health care provider, highlighting the acts of omission or interventions that may make the difference between a high risk pregnancy terminating without complication or ending as a near-miss or mortality. We reviewed the literature about various causes of maternal mortality and morbidity. In addition to the sincere efforts done by the doctors from different specialities, a good blood bank facility, ICU care as well as government provided transport facility played important roles in these cases. A long term analysis of this data can certainly guide the policy makers about the areas that need more stress and financial support.
\end{abstract}

Keywords: Maternal mortality, Near-miss case, Pregnancy

\section{INTRODUCTION}

A maternal near-miss case is defined as "a woman who nearly died but survived a complication that occurred during pregnancy, childbirth or within 42 days of termination of pregnancy". ${ }^{1}$ Practically, maternal nearmiss includes all the pregnancies where women survive life-threatening conditions (i.e. vital organ dysfunction). These patients share many pathological and circumstantial factors with the cases where there is death of a woman while pregnant or within 42 days of termination of pregnancy, irrespective of the duration and the site of the pregnancy, from any cause related to or aggravated by the pregnancy or its management, but not from accidental or incidental causes i.e. the cases of maternal mortality. ${ }^{1}$

One of the near-miss cases is of postpartum hemorrhage secondary to broad ligament hematoma leading to hemorrhagic shock; another case is that of sepsis leading to coagulopathy and ARDS. The other two cases are of indirect causes, one of them ending as a near-miss in a case of rheumatic heart disease with atrial fibrillation and the other one ending in maternal mortality was a case of pulmonary tuberculosis with respiratory failure. A combined study of maternal mortality and near-miss case can tell us the true incidence of pregnancy related life threatening conditions in a society and a comparison of two groups can guide us about the modifiable risk factors 
as well as the improvement possible in the quality of health care for obstetric population. A large scale audit over a long duration of period can truly guide an institute for the required interventions.

\section{REVIEW OF LITERATURE}

Ours is an obstetric unit of a tertiary care medical college in Punjab and a prospective study is underway since March 2015 of severe maternal outcome i.e. maternal near-miss cases and maternal deaths as defined by WHO with the objective of calculating important indicators like Maternal Near-Miss Ratio [MNMR], Severe Maternal Outcome Ratio [SMOR], maternal near-miss mortality ratio, mortality index and various process indicators as defined by WHO. ${ }^{1}$ Here we review the literature with typical cases as examples.

In addition to these indicators, each individual case is a lesson in itself of the various human errors committed at the level of patients, their families and the health care providers that can lead to transformation of a physiological event into a catastrophe.

\section{Table 1: WHO organ dysfunction based criteria.}

\section{Cardiovascular dysfunction}

Shock, cardiac arrest (absence of pulse/heart beat and loss of consciousness), use of continuous vasoactive drugs, cardiopulmonary resuscitation, severe hypoperfusion (lactate $>5 \mathrm{mmol} / \mathrm{l}$ or $>45 \mathrm{mg} / \mathrm{dl}$ ), severe acidosis $(\mathrm{pH}<7.1)$

\section{Respiratory dysfunction}

Acute cyanosis, gasping, severe tachypnea (respiratory rate $>40$ breaths per minute), severe bradypnea (respiratory rate $<6$ breaths per minute), intubation and ventilation not related to anaesthesia, severe hypoxemia $\left(\mathrm{O}_{2}\right.$ saturation $<90 \%$ for $=60$ minutes or $\left.\mathrm{PAO}_{2} / \mathrm{FiO}_{2}<200\right)$

\section{Renal dysfunction}

Oliguria non-responsive to fluids or diuretics, dialysis for acute renal failure, severe acute azotemia (creatinine $=300 \mu \mathrm{mol} / \mathrm{ml}$ or $=3.5 \mathrm{mg} / \mathrm{dl}$ )

\section{Coagulation/haematological dysfunction}

Failure to form clots, massive transfusion of blood or red cells ( $=5$ units), severe acute thrombocytopenia ( $<50000$ platelets/ml)

\section{Hepatic dysfunction}

Jaundice in the presence of pre-eclampsia, severe acute hyperbilirubinemia (bilirubin $>100 \mu \mathrm{mol} / \mathrm{l}$ or $>6.0 \mathrm{mg} / \mathrm{dl}$ )

\section{Neurological dysfunction}

Prolonged unconsciousness (lasting=12 hours)/coma (including metabolic coma), stroke, uncontrollable fits/status epilepticus, total paralysis

\section{Uterine dysfunction}

Uterine haemorrhage or infection leading to hysterectomy
We present here a case study of severe maternal outcomes (maternal near-miss cases and maternal deaths) at our obstetric care unit over a span of 5 weeks highlighting the variety of conditions that can put the life of a woman at risk during her pregnancy. In the period from $1 / 03 / 2015$ to $4 / 04 / 2015$, we had 161 deliveries, 150 live births and 11 patients were received with postpartum complications. 41 patients presented with potentially life threatening conditions of which 4 had a severe maternal outcome. There was one maternal mortality and 3 patients fulfilled the near-miss criteria of organ dysfunction as described by WHO (Table 1). ${ }^{1}$

\section{Case 1}

A postpartum patient presenting with hemorrhagic shock and coagulopathy due to postpartum broad ligament hematoma 28 years old, P3L3A0 rural resident was referred to us in emergency on 10/03/2015 with postpartum haemorrhage and shock following vaginal delivery of a term live baby $3.5 \mathrm{~kg}$ at $\mathrm{CHC}$. Patient had no history of antenatal checkups, no history of use of haematinics, 2 injections of tetanus toxoid had been taken and her antepartum haemoglobin report was 8 gram\%. It was an unsupervised pregnancy. She had got admitted at $\mathrm{CHC}$ on the same day with labour pains and had delivered around 4 hours before she was received at our hospital.

On examination, patient was confused, sedated and disoriented to surroundings. She was very pale with cold and clammy extremities. Her radial pulse was not palpable and blood pressure was 50/30 $\mathrm{mm} \mathrm{Hg}$, respiratory rate was $30 / \mathrm{min}$ and percentage saturation of oxygen $\left(\mathrm{SpO}_{2}\right)$ was $66 \%$. Abdomen was soft, non tender and uterus was 24 weeks in size and well contracted and retracted. There was dark altered blood coming from vaginal canal and a urethral catheter was already in situ with $50 \mathrm{cc}$. of haemorrhagic urine.

Immediate resuscitation was started with i.v. fluids and oxygenation and patient was shifted to OT for exploration of lower genital tract after sending samples for cross matching and haematological and biochemical investigations. On investigations, $\mathrm{Hb}=3.2 \mathrm{~g} \%, \mathrm{PTI}=23 \%$ and on clot retraction test, there was no clot formed in 30 minutes. Liver and renal tests were normal. Patient was intubated and put on mechanical ventilation and a quick lower genital tract exploration was done under all aseptic precautions. Dark non clotting blood was coming from uterine cavity and no placental bits or membranes were felt in the uterine cavity and haemostatic stitches were taken at a cervical tear at $10^{\prime} \mathrm{O}$ clock position, a vaginal laceration in the posterior vaginal wall and at a right sided para-urethral tear. Patient was transfused 4 units of blood and 8 units FFP. Patient was continued on ventilatory support and when bleeding did not stop after improvement in coagulation profile, she was taken for laparotomy taking the consent of family members for hysterectomy if required. 


\section{Peroperative findings}

Laparotomy was done under general anaesthesia by senior gynaecologist. On entering the peritoneal cavity, uterus was 24 weeks with lower segment markedly ballooned up by blood clots. A large hematoma was present in right broad ligament that was extending upwards till right fallopian tube and ovary and extending laterally in the retroperitoneal region. A decision for hysterectomy was taken and successive bilateral clamps were applied. A $2 \mathrm{X} 2 \mathrm{~cm}$ transverse rent was present in the right lateral edge of lower uterine segment. The clamp on right sided uterine vessels was carefully applied incorporating the rent and protecting the bladder and right ureter. Subtotal hysterectomy along with right sided oophorectomy was done. Patient had a cardiac arrest during surgery and cardiopulmonary resuscitation (CPR) was done by the anaesthetist. Complete haemostasis was assured, there was no vaginal bleeding, no bladder injury but hematuria was persistent. Abdomen was closed after inserting abdominal drain and patient shifted to intensive care unit (ICU).

Patient was transfused a total of 9 units blood, 19 fresh frozen plasmas, 4 random donor platelets and 2 units of cryoprecipitate during her management. Coagulopathy and hematuria slowly resolved over time. Patient again had a cardiac arrest on the third postoperative day and was revived with CPR. She was finally extubated on $4^{\text {th }}$ postoperative day and her chest physiotherapy was started. Patient had acute renal failure and haemodialysis was done on $3^{\text {rd }}$ and $9^{\text {th }}$ postoperative day. The catheter was removed after 2 weeks and there was no problem with wound healing. Patient was shifted from ICU on 16th postoperative day and was discharged on $28^{\text {th }}$ postoperative day.

\section{Case 2}

Elderly primigravida patient Mrs. M, 34 year old with 38.5 weeks pregnancy was referred to us from district hospital with acute respiratory distress for 12 days and leakage per vaginum for 16 hours on March $24^{\text {th }} 2015$.

On examination, patient was conscious, oriented, tachypnoeic with inability to complete a sentence due to dyspnoea. She had pallor and peripheral cyanosis. Pulse was 110/ $\mathrm{min}$, BP 110/60 mm $\mathrm{Hg}$, RR 40/min with widespread crepitations and rhonchi in the chest and decreased air entry on right side. She was in first stage of labour with absent membranes and fetal head at-3 station.

Patient gave history of tuberculosis in childhood after which she had been told that one of her lungs was severely damaged but no records were available of the same. She had breathlessness for 12 days for which she had been taking steroids and bronchodilators and had refused admission at district hospital 2 days back. She was HBsAg positive. Her arterial blood gas (ABG) showed increased $\mathrm{pCO}_{2}$.
Supportive treatment was started with oxygenation, nebulisation and antibiotics, anesthetist and physician consultation taken with plans to shift her to ICU after delivery. There was no progress in labour for four hours and she was taken up for LSCS due to worsening respiratory distress with labour pains. The surgery was uneventful and patient was shifted to ICU for ventilator support and further management. Patient's general condition improved in postoperative period. A chest x-ray confirmed right lung hypoplasia and cardiomegaly. On $8^{\text {th }}$ postoperative day, patient again developed respiratory distress; she was again intubated and put on ventilator. ECG revealed global ischemic changes. She had a cardiac arrest from which she could not be revived.

\section{Case 3}

Mrs. Y, 22 years old primigravida with 31 weeks twin gestation was admitted on March $31^{\text {st }} 2015$ with abdominal pain, distension, fever, jaundice and constipation for 10 days for which she was hospitalised at some private hospital for a week before being referred to us.

On examination, patient was conscious and oriented but severely distressed with abdominal pain which was only partially relieved with medications. Patient was poorly built, pallor and icterus were present. Patient was afebrile with a pulse rate of 120/min, BP $100 / 60 \mathrm{mmHg}$, RR 20/ min and no abnormality on cardiorespiratory examination. Abdomen was unduly distended, tense and tender with absent bowel sounds. Heart sounds of both fetuses were normal. Bowel sounds were sluggish.

USG showed two viable fetuses of 31 weeks gestation with oligohydramnios in one sac. Patient's gut was distended but no focal lesion could be identified which could have led to obstruction. Investigations showed a hemoglobin of $5.5 \mathrm{~g} / \mathrm{dl}$, total leukocyte count (TLC) equal to $15,500 / \mathrm{mm}^{3}$, total bilirubin $6.3 \mathrm{~g} \%$, liver transaminases were normal and prothrombin time index was $58 \%$. Her peripheral blood film was suggestive of anaemia of chronic disease. Thyroid function tests revealed hypothyroidism.

A provisional diagnosis of subacute intestinal obstruction with sepsis and anaemia was made and supportive treatment along with i.v. antibiotics was started pending the reports of blood and vaginal cultures. Patient's pain and distension worsened and a decision to perform LSCS was taken suspecting abruptio placenta.

During LSCS, there was ascites and peritoneal fluid was sent for chemistry and culture studies. Two live preterm foetuses were delivered, placenta delivered and uterus closed. There was no abruptio placenta. A thorough examination of abdominal cavity was performed by a general surgeon. No pathology was found in small and large intestines. A hard fecolith was present in the sigmoid colon. Abdomen was closed in layers. 
Postoperatively, patient started developing fever and abdominal distension from $3^{\text {rd }}$ postoperative day. Ascitic fluid examination revealed peritonitis [TLC $=4600 / \mathrm{cu}$ $\mathrm{mm}$, differential leukocyte count (DLC) was 90/10/0/0]. Urine and vaginal cultures showed growth of Klebsiella sp. Antibiotic therapy was continued as per culture sensitivity, 7 units blood and 7 units fresh frozen plasma were transfused for correction of anemia and coagulopathy. Patient developed acute respiratory distress syndrome (ARDS) on $15^{\text {th }}$ postoperative day and was shifted to ICU on ventilator support. She was extubated after 2 days. She had a full length wound gap which was resutured and her bowel function recovered. She was finally discharged on $29^{\text {th }}$ postoperative day.

\section{Case 4}

A 22 years old primigravida with 34 weeks twin gestation with placenta previa was referred from district hospital with chief complaints of bleeding per vaginum and palpitation on $3^{\text {rd }}$ April 2015. Patient had recurrent history of palpitation episodes throughout pregnancy. She had an episode of spotting through vaginal route but no labour pains. She had a ultrasonography (USG) one day back which showed diamniotic dichorionic pregnancy with first twin dead and second twin alive at 34 weeks gestation with transverse lie. Placenta was posterior, low lying and covering the internal os.

On examination, patient was conscious and well oriented. She was afebrile, pulse was $160 / \mathrm{min}$ irregularly irregular, BP 100/60 mm Hg, RR 16/min. Left precordium was heaving and a diastolic murmur was present. JVP was raised and there were crepitation in basal lung zones bilaterally. She was diagnosed as having rheumatic heart disease with atrial fibrillation and heart failure. Cardiologist consultation was taken and patient started on digoxin and penicillin along with supportive care and shifted to coronary care unit (CCU). Patient was not in labour and bleeding was minimal, coagulation profile was normal and so LSCS was planned to be done only after stabilisation of her condition. Echocardiography revealed a severe mitral stenosis with moderate mitral regurgitation with pulmonary arterial hypertension with left ventricular ejection fraction (LVEF) $50 \%$.

On $4^{\text {th }}$ day of hospitalisation, patient went into labour with a fresh episode of antepartum haemorrhage. Her pulse rate had stabilised at $100 / \mathrm{min}$ by that time. An emergency LSCS was done delivering a dead macerated fetus followed by a healthy preterm baby. A meticulously done surgery resulted in little blood loss and patient was shifted back to CCU for further management from where she was discharged after 10 days after complete counselling regarding her cardiac condition, need for continuous treatment, regular follow up and contraception. She was also advised for a detailed investigation before planning for next pregnancy.

\section{DISCUSSION}

The Millennium Development Goals (MDG) of the United Nations targeted to reduce by three quarters, between 1990 and 2015, the maternal mortality ratio. That means for India to target bringing down the estimated MMR of 1990 which was 437 per 1,00,000 live births to 200 maternal deaths per 1,00,000 live births by 2007 and 109 per $1,00,000$ live births by 2015 . $^{2,3}$ As per the latest estimates, the MMR status at all India level is at 174 in 2015 which means we have missed on the goal number 5 of MDG by a significant margin. ${ }^{3,4}$ India alone contributed to $15 \%$ of maternal mortality worldwide with approximately 45000 maternal deaths in 2015 . $^{4,5}$ Though, more than $50 \%$ reduction has registered in the number of maternal deaths in the last two decades, the present status shows that, even now, more than 120 women die of causes associated with pregnancy, in a day, in India! ${ }^{4,5}$ As per the Sample Registration System estimates of 2011-13, $68 \%$ of maternal deaths were in women in the age group of 20-29 years. $^{2}$

The maternal deaths being a rare event require prohibitively large sample size to provide robust estimates. Maternal mortality (MM) is frequently described as "just the tip of the iceberg" and maternal near-miss (MNM) is the much wider and largely ignored base of this icerberg. ${ }^{5-7}$ Reviews of MNM cases are likely to yield useful information as MNM cases are not as rare as maternal deaths and more honestly reported. They have the same pathways which lead to mortality and provide information regarding care received, acts of omission and possible means of prevention. ${ }^{8}$

Case 1 was a case of postpartum haemorrhage with haemorrhagic shock. Other than mild anaemia and the fact that she was not taking regular ANC; it was essentially not a high risk pregnancy and it was an institutional delivery.

The delivery had been conducted at a CHC and we were not aware whether third stage was actively managed, the refer slip didn't mention about any instrumental delivery and the neonate was alive and healthy. The uterus was well contracted and lower genital tract exploration had been done. The bleeding was uterine and when replacement of clotting factors didn't stop bleeding, laparotomy was decided suspecting an upper genital tract trauma.

Broad ligament hematoma following a vaginal delivery is rare with incidence quoted as low as 1:20,000 and few cases have been reported. Broad ligament hematoma results from a tear in the upper vagina, cervix, or uterus that extends into uterine or vaginal arteries. ${ }^{9,10}$ Abdominal pain may be the only symptom and patient often presents with shock with or without vaginal bleeding. A high index of suspicion is required to diagnose and manage these haematomas promptly before signs of cardiovascular collapse develop. On abdominal 
examination the uterus is deviated upward and laterally, to the opposite side from the broad ligament haematoma. ${ }^{11}$ Ultrasound may help in diagnosis, otherwise it is almost a diagnosis of exclusion when uterus is not atonic and there are no cervical, vaginal or perineal tears and signs of shock or hemorrhage persist or worsen.

Small hematomas may be managed conservatively in stable patients but conservative management of larger haematomas has been associated with longer stays in hospital, an increased need for antibiotics and blood transfusion and greater subsequent operative intervention. ${ }^{12}$ Surgical intervention carries the risk of injury to surrounding viscera especially ureters. Pelvic vessel embolisation may be used as the first line treatment for persistent bleeding, but the main limitation of the wider use of interventional radiology for postpartum haemorrhage is the limited availability of expertise and equipment in most units. ${ }^{13,14}$

Our patient was bleeding, in shock and the hematoma was also extending laterally in the retroperitoneal space. She could be saved because of timely referral, government provided transport facility, availability of blood and blood components at the tertiary centre, a timely decision for laprotomy and hysterectomy at the hands of experienced surgeon and good anaesthetist and ICU care.

Haemorrhage was the leading direct cause of maternal death worldwide, representing $27.1 \%$ of maternal deaths between 2003 and 2009. ${ }^{15}$ More than two thirds of reported haemorrhage deaths were classified as postpartum haemorrhage. ${ }^{15}$

At an educational institute, this case was a good reminder for all residents that the labour of a third gravid patient needs to be monitored in all stages just like that of a primigravida. It was not exactly a preventable condition but her survival after severe shock, coagulopathy, two episodes of cardiac arrest, acute renal failure was a result of teamwork of different specialities as well as a functional referral system and advanced blood bank facilities.

Case 2 and 4 were both cases of pre-existent medical diseases that had worsened with the progress of gestation and/or labour pains. Both the patients had been taking regular antenatal check-ups and had been fairly symptomatic during the course of pregnancy. Unfortunately, their serious medical disease was not suspected, investigated or documented by any of the health care providers. A basic general examination including cardiovascular examination would have resulted in timely diagnosis and intervention.

Case 2 was a case of chronic respiratory disease with right pulmonary hypoplasia and history of treatment taken for tuberculosis. Patient had hidden the history from her family and ANC provider perhaps due to social stigma attached with tuberculosis. Worldwide, TB kills more women each year than any other infection and the greatest burden of disease occurs in those in the reproductive age (15 to $49 \mathrm{y}$ old). ${ }^{14}$ There was history of refusal of hospitalisation 2 days back which further delayed the treatment. The LSCS was uneventful but she died on $8^{\text {th }}$ postoperative period due to peripartum cardiomyopathy. The condition was not preventable but a timely diagnosis and intervention could have prevented the mortality.

Case 4 had atrial fibrillation and LVF secondary to an undiagnosed rheumatic heart disease. Simultaneously there were obstetric risk factors of placenta previa, twin gestation and intrauterine demise of one of the twins. Fortunately her cardiac failure and atrial fibrillation were managed within 3 days of her admission when an emergency LSCS had to be done due to antepartum haemorrhage and preterm labour. Her medical condition continues to be a risk factor for all her future obstetric events for which a detailed counselling of patient and her family was done.

Indirect obstetric deaths are those resulting from previous existing disease or disease that developed during pregnancy and which was not due to direct obstetric causes, but which was aggravated by physiologic effects of pregnancy. ${ }^{16}$ Indirect causes include infections (e.g., malaria and hepatitis), cardiovascular disease, psychiatric illnesses (e.g., suicide and violence), tuberculosis, epilepsy, and diabetes. A combination of rising prevalence of non-communicable chronic conditions such as cardiovascular disease and diabetes and already prevalent chronic infectious diseases like tuberculosis, HIV and malaria are increasingly contributing to indirect maternal deaths. Between 2003 and 2009, more than a quarter of maternal deaths globally were attributable to indirect causes. ${ }^{15}$ This has implications for intervention strategies beyond emergency obstetric care where indirect causes kill many women of reproductive age. Despite the importance of these indirect causes, key policy and strategy documents of leading international maternal health nongovernmental organisations and UN organisations do not focus much on indirect causes of maternal mortality, except for HIV infection. ${ }^{16}$ Predominant attention is still given to direct causes of adverse pregnancy outcomes.

In spite of all components of comprehensive emergency obstetric care, patient 2 could not survive and an important contributor to the survival of patient 4 was her timely diagnosis with echocardiography and CCU care before operative intervention. This is possible only at the level of a tertiary care centre but there also these facilities are not available round the clock due to shortage of specialist doctors.

Case 3 was a case of sepsis with sub-acute intestinal obstruction of a malnourished anaemic mother with twin 
gestation. Exact site or source of infection and the pathogen could not be identified but patient's anaemia and twin gestation were the known risk factors for her decreased immunity. Sepsis led to peritonitis, coagulopathy, electrolyte disturbances, ARDS and wound dehiscence thus increasing the maternal morbidity. She was saved because of antibiotics and supportive therapy along with a good blood bank and ICU helping us. Sepsis is the direct obstetric cause of $10.7 \%$ deaths in 2003-09. ${ }^{15}$

In the remaining 37 patients presenting with potentially life threatening conditions, there were 14 cases of severe preeclampsia and 4 of eclampsia. An almost universal use of magnesium sulphate even in peripheral centres, timely referral, transport facility provided by government and a policy of timely delivery has contributed to the fact that none of these patients suffered organ dysfunction defining near-miss mortality. This is a success story for the EmOC services when hypertensive disease is responsible for $14 \%$ of maternal mortality. ${ }^{16}$ Similarly 2 cases of ruptured ectopic pregnancy, 4 cases of incomplete abortion with severe anemia, 7 antenatal patients with severe anaemia and 4 cases of postpartum haemorrhage were managed timely with timely EmOC and blood bank facilities. There were 2 patients with medical diseases (one with jaundice and one with pneumonitis) that were managed in coordination with physician.

The maternal near-miss approach is not just helpful in guiding us about the condition of maternal health and the process indicators of the health services; each case in itself is a lesson in critical obstetric care. These lessons will guide us to target 3.1 of SDG 3 to reduce the global MMR to less than 70 per 100,000 live births by 2030 which needs immense dedication and some serious innovations in our healthcare policies. ${ }^{8}$

\section{CONCLUSION}

Identifying all potential cases is the key to successful execution of this strategy. To make sure that all such cases are successfully recorded, clear cut criteria should be defined for inclusion, the treating doctors should appropriately tabulate the data after proper assessment and report them. Health care providers must foresee such complicated pregnancies so that they can reduce poor maternal outcomes with early interventions.

A discussion and reporting of these cases along with cases of maternal mortality can guide us about the pitfalls in care, interventions needed to prevent them and also, in many cases can help us appreciate the good work being done by health care providers in a society that is keen on projecting a negative image of this noble profession.

Funding: No funding sources Conflict of interest: None declared

Ethical approval: Not required

\section{REFERENCES}

1. World Health Organization [Internet]. Evaluating the quality of care for severe pregnancy complications: the WHO near-miss approach for maternal health. [Updated 2011 July; accessed on 2015 Aug] Available from: http://apps.who.int/iris/ bitstream/10665/44692/ 1/9789241502221_eng.pdf

2. Office of Registrar General India [Internet]. Special Bulletin on Maternal Mortality in India 2007-09 Sample Registration System (SRS) office of Registrar General, India, June 2011. [Updated on: 2011 Jun 1; accessed on 2015 Sep 15] Available from: http://censusindia.gov.in/ vital_statistics/ SRS_Bulletins/Final-MMR\%20Bulletin-200709_070711.pdf

3. Ministry of Statistics and Programme Implementation [Internet]. Millennium Development Goal India Country Report, Social Statistics Division, Ministry of Statistics and Programme Implementation, Government of India. [Updated: 2015 February 24; accessed on 2015 Aug] Available from: http://mospi.nic.in/Mospi_New/ upload/ mdg_26feb15.pdf

4. Patel V, Rahman A, Jacob KS, Hughes M. Effect of maternal mental health on infant growth in low income countries: new evidence from south Asia. BMJ. 2004;328:820-23.

5. World Health Organization [INTERNET]. Strategies towards ending preventable maternal mortality (EPMM). Geneva. 2015. (Updated: 2015 November 5). Available from: http:// www.everywomaneverychild.org/ images/ EPMM_final_report_2015.pdf

6. World Health Organization [INTERNET]. Trends in Maternal Mortality: 1990 to 2015. Available from: http://apps.who.int/iris/bitstream/ 10665/ 194254/ 1/ 9789241565141_eng.pdf.

7. Kassebaum NJ, Bertozzi-Villa A, Coggeshall MS, Shackelford KA, Steiner C, Hueton KR. Global, regional, and national levels and causes of maternal mortality during 1990-2013: a systematic analysis for the Global Burden of Disease Study 2013. Institute for Health Metrics and Evaluation. Lancet. 2014;384(9947):980-1004.

8. Purandare CN. Maternal near-miss review: a way forward. Journal of Obstetrics and Gynaecology of India. 2013;63(4):213-5.

9. Saleem N, Ali HS, Irfan A, Afzal B. Broad ligament hematoma following a vaginal delivery in primigravida. Pakistan Journal of Medical Sciences. 2009;25(4):683-5.

10. Murali R, Hosni MM, Navaneetham N. A Rare Case of Broad Ligament Haematoma 24 Hours after Normal Vaginal Delivery. J Clin Case Rep. 2014;4(7):391.

11. Mawhinney S, Holman R. Puerperal genital haematoma: a commonly missed diagnosis. The Obstetrician \& Gynaecologist. 2007;9:195-200. 
12. Chin HG, Scott DR, Resnik R, Davis GB, Lurie AL. Angiographic embolization of intractable puerperal haematomas. Am J Obstet Gynecol. 1989;160:434-8.

13. Bloom AI, Verstandig A, Gielchinsky Y, Nadiari M, Elchalal U. Arterial embolisation for persistent primary postpartum haemorrhage: before or after hysterectomy? BJOG. 2004;111:880-4.

14. Say L, Chou D, Gemmill A, Tunçalp Ö, Moller AB, Daniels J, et al. Global causes of maternal death: a WHO systematic analysis. Lancet Glob Health. 2014;2(6):e323-33
15. Nhan-Chang CL, Jones TB. Tuberculosis in Pregnancy. Clinical Obstetrics and Gynecology. 2010;53(2):311-21.

16. Torm F, Agampodi S, Eddleston M, Sørensen JB, Konradsen F, Rheinländer $\mathrm{T}$. Indirect causes of maternal death. The Lancet Global Health. 2014;2(10):e566.

Cite this article as: Grover S, Shergill HK, Chhabra A. Severe maternal outcome: a review. Int J Reprod Contracept Obstet Gynecol 2016;5:596-602. 